\title{
COMPARACIÓN DE VOZ BAJO EL COCIENTE DE PROBABILIDAD EN EL CASO DE LUIS TRALCAL (2011)*
}

\author{
VOICE COMPARISON FROM LIKELIHOOD RATIO \\ IN LUIS TRALCAL'S CASE (2011)
}

\section{CLAUDIA ROSAS AGUILAR}

UACH, Universidad Austral de Chile. Valdivia, Chile

claudiarosas@uach.cl

JORGE SOMMERHOFF HYDE

UACH, Universidad Austral de Chile. Valdivia, Chile

sommerhoff@uach.cl

CÉSAR SÁEZ ELGUETA

PDI, Policía de Investigaciones de Chile

cesarsaez.e@gmail.com

SEBASTIÁN SAAVEDRA CEA

Centro de Investigación y Defensa Sur

ssaavedracea@gmail.com

\section{RESUMEN}

A partir de un caso que involucró en el 2011 a los autores, se comparan la muestra de habla de Luis Tralcal, sospechoso de amenaza terrorista, calificada por el Ministerio Público -obtenida a partir de una declaración de audiencia- y la muestra incriminatoria -extraída de una reunión grabada, realizada entre dirigentes mapuches y un delegado de la CONADIcon el fin de analizar científicamente si pertenecen al mismo locutor -lo que daría apoyo a la hipótesis de la Fiscalía- o a locutores diferentes -lo que daría apoyo a la hipótesis de la Defensa. Para ello, se aplica el método de cálculo del cociente de probabilidad (Likelihood ratio, LR) que permite evaluar numéricamente las diferencias de los parámetros de

\footnotetext{
* Queremos agradecer el consentimiento informado, libre y espontáneo de Luis Tralcal, imputado en su momento en el caso referido, no solo por permitir que su nombre apareciera publicado en este trabajo -a pesar del coste personal y familiar que esto pudiera implicar-, reforzando con ello la veracidad del estudio realizado, sino también, por comprender y desear que se comparta un enfoque científico como el utilizado, en pro del resguardo de cualquier persona involucrada en delitos de voz. Igualmente, es necesario agradecer el financiamiento específico otorgado a través del Fondecyt Regular 1110742, que ha permitido desarrollar esta investigación, sin el cual nada de esto habría sido posible.
} 
comparación seleccionados entre las muestras del sospechoso y del delincuente bajo la probabilidad de que proceden del mismo locutor y la probabilidad de que proceden de locutores diferentes, teniendo en cuenta la población de referencia. Para la comparación se utilizó el F2 del fonema /a/ de las muestras en disputa a la luz de dos tipos de población de referencia: las muestras de 4 hablantes presentes en el audio de la CONADI y luego, las muestras de 25 hablantes mapuches, extraídas de Internet. En ambos casos, los resultados del cociente de probabilidad dan apoyo a la Defensa.

Palabras clave: Cociente de probabilidad, comparación forense de voz.

\begin{abstract}
From a case where the authors were involved in 2011, the sample voice of Luis Tralcal are compared, suspect of terrorist threat, as qualified the Public Ministry -obtained from a statement incriminating and hearing- and the incriminating speech sample, extracted from a recorded meeting, held between Mapuches leaders and a delegate from the CONADI -in order to analyze scientifically whether they belong to the same speakerwhich would support the prosecution hypothesis -or to different speakers- which would support the defense hypothesis. For this, the likelihood ratio calculating method (LR) that allows the evaluation of the numerical difference of the comparing parameters selected from the samples of the suspect and offender under the probability that they belong to the same speaker and the probability that they belong to different speakers, considering the reference population. For comparison, the F2 of the phoneme /a/ of the samples in dispute were used in the light of two population reference types: the samples of 4 speakers present in the CONADI audio and then, the samples of 25 Mapuche speakers extracted from Internet. In both cases, the likelihood ratio results give support to the defense.
\end{abstract}

Keywords: Likelihood ratio, forensic voice comparison.

Recibido: 15/11/2013. Aceptado: 16/04/2014.

\title{
1. ANTECEDENTES GENERALES
}

\subsection{El caso: La acusación de amenaza terrorista}

Zl caso se remonta al año 2008 y tiene relación con las negociaciones de coUmuneros mapuches del lof Lleupeco, comuna de Padre Las Casas, IX Región, quienes reivindican tierras ocupadas por colonos chilenos y extranjeros y que les fueran entregadas por el Estado de Chile tras la ocupación militar de la Araucanía llevada a cabo durante la segunda mitad del siglo XIX. En tal contexto, se llevaron a cabo una serie de reuniones entre dirigentes mapuches y personeros de la 
CONADI, con el objeto de llevar a cabo un proceso de compra de las tierras ancestralmente ocupadas por las comunidades indígenas. Tras una serie de reuniones que se prolongaban por más de dos años, sin una respuesta precisa por parte de los órganos del Estado, los dirigentes increparon la forma de actuar de la CONADI, lo cual quedó grabado en un registro de audio MP4 que con fecha 22 de enero de 2009 se incautó desde el domicilio de M.H.H ${ }^{1}$. Al revisar dicho elemento, los funcionarios policiales escucharon la grabación de una reunión donde se manifestaba la molestia por la demora en la compra de tierras reivindicadas. Los oficiales investigadores dan cuenta de un delito de amenaza, el cual es calificado de terrorista por el Ministerio Público. Asimismo, tanto los funcionarios de la PDI como los fiscales del Ministerio Público señalan que algunas de las voces contenidas en la grabación del MP4 corresponden a los acusados Tralcal y M.H.H., dirigentes tradicionales de las comunidades mapuches del sector.

\subsection{El informe pericial y la absolución del imputado}

La defensa de Luis Tralcal presenta como prueba de descargo a los peritos Jorge Sommerhoff y Claudia Rosas. En ese contexto, ambos peritos elaboran un informe que responde a la solicitud hecha por el abogado de la Defensa, Sebastián Saavedra, para determinar si la muestra de discurso del sospechoso Luis Tralcal y del audio de la CONADI proceden del mismo hablante o de hablantes diferentes.

El Informe, que se divide en dos partes, expone en primer lugar las dificultades que enfrenta la ciencia forense actual para determinar con un 100 por ciento de certeza la culpabilidad o inocencia de una persona, a través del cotejo de muestras de habla de un sospechoso y de un delincuente, debido, entre otras varias razones, a la enorme variabilidad intralocutor. Luego, tras una serie de consideraciones técnicas sobre el formato de las muestras disponibles para el peritaje, el Informe presenta los resultados de un análisis de los parámetros acústicos de F0, F1 y F2 entre las muestras de discurso del sospechoso y del delincuente, con el fin de respaldar con datos empíricos la variabilidad comentada y apoyar la tesis que impide su valor probatorio.

Finalmente, el Tribunal decidió absolver a ambos acusados, teniendo en cuenta -además de otros elementos- la variabilidad de la voz referida en el informe pericial emitido y refrendado en la declaración del perito Sommerhoff y de su propia experticia $^{2}$.

\footnotetext{
${ }^{1}$ Se omite el nombre del involucrado para proteger su identidad.

${ }^{2}$ Causa RIT 194-2011 del Tribunal Oral en lo Penal de Temuco. Sentencia de fecha 8 de octubre de 2011. Considerando Cuadragésimo Tercero.
} 


\subsection{La investigación propuesta basada en un enfoque científico}

En esta oportunidad, quienes suscriben se proponen aplicar una metodología científica para comparar las muestras del caso mencionado en el marco de una línea de investigación forense de la voz iniciada hace ya algunos años ${ }^{3}$, con un doble propósito: primero, conocer cuál habría sido el resultado de la comparación de las muestras de habla en disputa de haberse aplicado en el caso de Tralcal (2011) una metodología como la que hoy se pretende utilizar; a la vez que servir de ejemplo de una forma de proceder y de los requisitos que supone su propia implementación para ser conocidos y debatidos por los jueces, abogados y peritos de Chile. En segundo lugar, también esperamos demostrar mediante este trabajo la importancia que supone para los involucrados en delitos de voz contar con un sistema de análisis para la comparación de la evidencia más objetivo, que otorgue mayor resguardo a las víctimas y más apoyo a los tribunales.

\section{MARCO TEÓRICO}

Desde la perspectiva de quien realiza la identificación forense de un hablante, existen dos tipos básicos: aquél donde quien la aplica es un testigo auditivo común y aquel otro donde quien actúa es un experto al que le son llevadas grabaciones del criminal y del sospechoso para determinar científicamente si proceden o no del mismo hablante. Este es el escenario que ahora nos interesa. A continuación se describirán algunos aspectos jurídicos para contextualizar el ejercicio pericial de la voz en Chile y luego se describirá el enfoque metodológico del tratamiento de la evidencia forense de voz que se utiliza en el presente trabajo.

\subsection{Aspectos jurídicos}

Conviene señalar que la reforma procesal penal ha sido uno de los cambios más importantes en la administración de la justicia chilena del último centenario, lo que ha implicado la sustitución de un sistema de raigambre inquisitivo por otro de corte acusatorio formal genuinamente contradictorio que satisfaga las exigencias del debido proceso. Esta nueva lógica obliga a reestructurar y replantear la forma

\footnotetext{
3 Se ha ido desarrollando una línea de investigación forense de la voz, formalizada a través de varios proyectos, Ref.: DID-UAChS-2005-72, "Estudio fonético-acústico de la variabilidad idiofónica en español para validar análisis periciales de voces presentadas en los juicios", 2005-2006; Fondecyt Regular 1070210, "Pautas para el análisis pericial de voces presentadas en los juicios, 2007-2010"; y Fondecyt Regular 1110742, "Voces en contextos periciales para el reconocimiento automático", 2011-2014.
} 
de hacer valer en juicio los medios probatorios por medio de los cuales se probará los hechos, materia de una acusación criminal.

En lo que dice relación con la prueba pericial, vale la pena resaltar el cambio que implica la adopción de un sistema acusatorio, tanto como los valores y principios que lo guían. Al respecto, la principal característica bajo la cual se estructuraba la prueba pericial en el antiguo sistema procesal era que los peritos eran concebidos, principalmente, como auxiliares de la administración de justicia, dentro de un sistema inquisitivo, fundamentalmente, escrito y secreto, bajo un sistema de prueba tasada, altamente regulada. Coherente con lo anterior, la prueba pericial estaba constituida por el informe pericial.

Dicha lógica cambia radicalmente en el nuevo sistema. En efecto, en el actual sistema procesal penal la prueba pericial imbuida por los principios de la publicidad, oralidad y contradictorio, está constituida por la declaración del perito en estrados. La prueba consiste en la declaración que en el juicio presenta el experto, sin que ella pueda ser reemplazada, sustituida o complementada por declaraciones previas registradas en actas o por su informe pericial escrito, salvo los casos excepcionales regulados por el propio Código Procesal Penal.

En cuanto a la valoración de la prueba, se establece un sistema de apreciación conforme a la sana crítica, es decir, los medios de prueba deben ser ponderados por el tribunal con libertad, teniendo sólo como límite las reglas de la lógica, las máximas de la experiencia y los conocimientos científicamente afianzados (Art. 297,CPP, 2010: 205-2006), esto es, los saberes cientificos y técnicos más o menos generalizados, comunes y compartidos como verdaderos por la gran mayoría de las personas que profesan una disciplina (Cerda, 2008: 39); de tal forma que los jueces al momento de valorar la prueba y determinar, en definitiva, acerca de la absolución o condena de una persona determinada deben respetar lo que la comunidad científica, mediante el método cientifico, ha logrado establecer como parámetro de realidad.

Ahora bien, la manera de acreditar ante el tribunal esta realidad científica, o el estado actual del arte u oficio determinado, deberá concretarse mediante la declaración en juicio del perito respectivo. Para ello, cada una de las partes deberá acreditar la calidad de su prueba pericial, mediante la exposición de los antecedentes curriculares de cada perito y de su experticia en el tema específico por el cual comparece, así como el rigor científico de sus conclusiones (Art. 318, CPP, 2010: 218). La discrepancia que exista entre dos peritos será decidida por la calidad y/o experiencia que logren acreditar las partes.

En cuanto a la prueba de voz, la manera de acreditar la correspondencia de una voz dubitada (en adelante, del delincuente o incriminatoria) con las de algún acusado, testigo y/o víctima se realizará mediante el respectivo peritaje de audio y sonido.

Como en todos los casos, el peritaje de audio y sonido debe cumplir los requisitos comunes a todo peritaje, esto es, que debe describir la cosa u objeto pericia- 
do, señalar las operaciones practicadas y, finalmente, indicar las conclusiones a las causas que ha arribado. Con respecto al habla, determinar si la voz incriminatoria corresponde o no a la voz indubitada (en adelante, del sospechoso).

Singular importancia tendrá al efecto la forma en cómo se obtuvo la voz del sospechoso; principalmente, cuando lo que se intentará acreditar es la culpabilidad de alguna persona en un delito; por cuanto, en virtud del principio de no autoincriminación, nadie se encuentra obligado a colaborar con su culpabilidad. En consecuencia, nadie puede ser obligado a colaborar en recoger evidencia en su contra, lo que implica que el imputado no puede ser obligado por los órganos de persecución criminal a someterse a una toma de muestra de voz sin su consentimiento. Cualquier contravención a estas normas implicará la exclusión del peritaje por haberse infringido garantías en su obtención, impidiendo su rendición en el juicio oral respectivo.

Por último, la demanda de informes periciales de voz producto de la reforma procesal penal comentada influyó notablemente en el aumento de la demanda de las autoridades judiciales respecto de la necesidad de emitir informes periciales de cotejos de voces. Por ello, la Policía de Investigaciones de Chile (PDI), que ha demostrado un interés permanente y creciente por los espacios necesarios a fin de desarrollar su trabajo con mayor rigurosidad y fiabilidad, en el año 2003 crea la Sección de Sonido, la que a partir de 2005 pasa a llamarse Sonido y Audiovisual, y recibe a la fecha la mayor cantidad de solicitudes de comparación en nuestro país ${ }^{4}$.

\subsection{Enfoque metodológico bayesiano}

En la determinación de la procedencia de la evidencia hay dos funciones bien diferenciadas que conviene explicitar: la función del juez y la función del perito. En cuanto al primero ya se sabe que su función será establecer la culpabilidad o inocencia de un sospechoso; mientras que al experto le corresponde colaborar en la decisión del juez en un sentido u otro, mediante su opinión informada. Su ámbito de acción se restringe, por tanto, a evaluar la evidencia, o más técnicamente, como se verá en este apartado, determinar las probabilidades de la evidencia. La razón de esto no sólo tiene que ver con una disposición legal, sino también, y sobre todo, con una cuestión lógica: es imposible que el experto forense pueda concluir en base a una sola evidencia la culpabilidad o inocencia de un acusado.

\footnotetext{
${ }^{4} \mathrm{La}$ información relacionada con los cambios estructurales experimentados por la PDI, producto de la Reforma Procesal Penal, consta en el "Proyecto para la creación de la Sección Sonido y Audiovisual del Laboratorio de Criminalística Regional Temuco", año 2008 (uso interno). Con respecto a los datos de las solicitudes de cotejos: según registros internos, en el año 2013 se recibió aproximadamente 87 solicitudes de comparación de voz (entre las secciones de Lacrim Central en Santiago y Lacrim de Temuco).
} 
Para poder hacerlo debería contar con toda la información disponible (evidencias de voz, de sangre, etc.).

Actualmente, se concibe que el perito forense, con el objeto de comparar las muestras incriminatorias y del sospechoso, aborde el análisis asumiendo un enfoque bayesiano que se basa en una relación de probabilidades, conocido como cociente de probabilidad, explicada ampliamente en Rose (2002). En los siguientes párrafos se expondrán los conceptos básicos que permitan comprender su lógica, estructura y funcionamiento para la interpretación de la aplicación al caso real que constituye el objeto de este trabajo.

\subsection{El cociente de probabilidad}

El concepto de cociente de probabilidad alude a una relación de probabilidades contrarias: la probabilidad de encontrar cierta evidencia asumiendo la hipótesis de que las muestras comparadas provienen de una persona y la probabilidad de observar la evidencia, asumiendo la hipótesis de que provienen de personas diferentes. En inglés el término es Likelihood ratio y se abrevia $L R$. Debido al alto grado de difusión del término, en la presentación de las fórmulas, conservamos los nombres de la lengua original.

Para comprender la lógica de este planteamiento, Rose (2002: 57) cita un ejemplo de Robertson y Vignaux (1995: 13) donde la Corte quiere averiguar si una niña en particular que se comía las uñas ha sido objeto de abusos sexuales en un contexto donde se sabe que un alto porcentaje (80\%) de la población de niños abusados sexualmente se comen las uñas, lo que deja de manifiesto que para evaluar la evidencia se requiere también conocer el porcentaje de niños que comparten la evidencia (comerse las uñas) y que no han sido abusados. Si este porcentaje es menor que el de los niños abusados sexualmente -supongamos, 10 contra 80-, entonces la evidencia de comerse las uñas ofrecerá cierto apoyo a la hipótesis de abuso sexual; por el contrario, si es mayor, la evidencia de comerse las uñas no puede probar que la niña ha sido abusada sexualmente.

En fonética forense, esto normalmente equivale a estimar cuánto más probable es que sea el grado de similitud entre uno o más rasgos acústicos observados -supongamos un valor de $800 \mathrm{~Hz}$ y $820 \mathrm{~Hz}$ para el F1 del fonema /a/- entre las muestras de discurso incriminatoria y del sospechoso, respectivamente, si fueron dichas por el mismo locutor que por diferentes locutores.

\subsubsection{Sus componentes: la similitud, la tipicidad y la población de referencia}

El cociente de probabilidad requiere de la enunciación de tres componentes de información: la similitud, la tipicidad y la población de referencia. La similitud tiene 
que ver con el grado en que los valores de las muestras en disputa son semejantes -o menos diferentes-; y la tipicidad, con la probabilidad de encontrar ese mismo resultado (semejanza o diferencia) en cualquier par de muestras extraídas al azar de la población de referencia o relevante, que es el grupo de locutores de donde procede el sospechoso.

Para ilustrar su importancia, Rose (2002: 306-310) presenta un par de casos en los que las muestras comparadas difieren en la misma pequeña cantidad, por lo cual son muy similares; sin embargo, en uno, esa diferencia se ubica en el extremo inferior de la distribución de los valores de la población de referencia y en otro, dentro de su media. En el primer caso se trata de una combinación no típica y que al ser también similar ofrece un alto valor probatorio; mientras que en el segundo caso, la combinación es típica, dado que es posible encontrarla en cualquier par de la población, y por lo mismo no ofrece valor probatorio, pese a ser el resultado muy similar.

A la luz de estos ejemplos, queda de manifiesto que la magnitud de la diferencia entre las muestras del delincuente y del sospechoso -el concepto de similitud- no es suficiente por sí mismo; también se tiene que tomar en cuenta la tipicidad de las dos muestras evaluadas bajo la estadística de una población de referencia. Su perfil dependerá de cada caso; en particular, dependerá de la naturaleza de la hipótesis de la Defensa (también llamada hipótesis alternativa). Por ejemplo, en la descripción del caso de Tralcal (2011) que nos ocupa, la hipótesis de la Defensa podría ser "era la voz de otro (de otro hombre mapuche) distinto al acusado" o con mayor precisión: "era la voz de algún otro hombre mapuche que suena como el acusado". Bajo la primera hipótesis, la muestra de referencia corresponderá a todos los otros locutores mapuches varones; bajo la segunda, en cambio, solamente incluirá los locutores mapuches varones que suenan como el acusado.

El Cociente de Probabilidad (LR) puede expresarse de acuerdo a la fórmula, tal como aparece en la Fig. 1, de (Evett, 1991: 12) referida por Rose (2002: 58), donde LR es el cociente de probabilidad; $p$ es probabilidad; $E$, evidencia; $H_{p}$, hipótesis del fiscal $\left({ }_{p}\right)$ por el inglés prosecution); $\mathrm{y}_{\mathrm{d}}$, hipótesis de la Defensa.

$$
\mathrm{LR}=\frac{p\left(\mathrm{E} \mid \mathrm{H}_{\mathrm{p}}\right)}{p\left(\mathrm{E} \mid \mathrm{H}_{\mathrm{d}}\right)}
$$

Figura 1. Fórmula para el cociente de probabilidad.

Queda claro, entonces, que el cociente de probabilidad es el resultado del cálculo entre las dos probabilidades: la probabilidad de hallar la evidencia, bajo la hipótesis de la Fiscalía de que las muestras del delincuente y del sospechoso proceden del 
mismo locutor, o, lo que es lo mismo, que son más típicas de encontrar en un mismo locutor; versus la hipótesis de la Defensa de que la evidencia observada es más típica de encontrar entre locutores diferentes, es decir, en cualquier combinación de muestras de la población de referencia, obtenida al azar.

\subsubsection{La magnitud del cociente de probabilidad}

Con respecto al resultado, cuanto mayor sea la diferencia entre las muestras del delincuente y del sospechoso, más pequeño se hace el numerador, y viceversa. Así, valores del cociente superiores a la unidad dan apoyo a la hipótesis del fiscal; mientras que valores del cociente inferiores a la unidad dan apoyo a la hipótesis de la Defensa. La cantidad de apoyo en cada caso es proporcional al tamaño del LR. Conviene destacar que un valor unitario del cociente es equívoco; implica que el analista tiene las mismas probabilidades de observar la evidencia si el acusado es culpable como si no lo es. Por lo tanto, no distingue entre las hipótesis de la Defensa y de la Fiscalía y no tiene valor probatorio.

Por otra parte, el número de observaciones en una muestra afecta los límites de confianza que se correlaciona con la magnitud del cociente; de manera que mientras más pequeño es el tamaño de la dimensión de las dos muestras que se están comparando, más tiende el cociente hacia la unidad. Así, el número de observaciones en una muestra se traduce muy bien en la solidez de la evidencia.

\subsubsection{El cociente de probabilidad y su propiedad combinatoria}

Una de las ventajas del enfoque del cociente de probabilidad (LR) para el análisis de la voz es su propiedad de combinarse con otros (cocientes de probabilidad). Esto quiere decir que es posible comparar muestras de voz bajo varios rasgos (F0, distintos formantes) de manera independiente y combinar los resultados de cada cálculo en otro momento y obtener un Cociente de probabilidad Global (Cfr. Robertson y Vignaux 1995: 69-72). Esto está simbolizado en la Fig. 2, donde $\operatorname{OLR}_{f-p}$ significa cociente de probabilidad fonético forense global; $\mathrm{LR}_{f-p} 1$ representa al cociente de probabilidad para la primera pieza de evidencia fonética forense $\mathrm{E}_{f-p} 1$; LR $\mathrm{E}_{f-p} 2$ para el LR del segundo elemento de evidencia, y así sucesivamente.

$$
\mathrm{OLR}_{f-p}=f\left(\mathrm{LRE}_{f-p} 1, \mathrm{LRE}_{f-p} 2, \ldots, \mathrm{LRE}_{f-p} \mathrm{~N}\right)
$$

Figura 2. Cociente de Probabilidad Global para distintas piezas de evidencias fonéticas forenses. 
El cociente de probabilidad combinado (OLR) es el producto de sus cocientes (LRs) asociados. Así, por ejemplo, dado un cociente de probabilidad (LR) de 3 de una pieza de evidencia fonética forense, y un LR de 4.2 de una segunda pieza de evidencia independiente de la primera, el cociente combinado es $(3 \times 4,2=)$ 12,6.

\subsubsection{Presentación de los resultados numéricos del cociente de probabilidad}

En pro de hacer más comprensibles los resultados numéricos del cociente de probabilidad se han propuesto dos tipos de escalas para expresar los resultados, si bien no existe consenso: la escala verbal y la escala logarítmica. A continuación se presentan ambos tipos extraídos de Champod y Evett (2000: 240-242), citados por Rose (2002: 61-62).

\section{a) Escalas verbales para el cociente de probabilidad}

Frente a la manifiesta claridad de una expresión verbal como ésta existe al mismo tiempo el peligro de que la ambigüedad propia del significado pueda prestarse para interpretaciones sesgadas por las partes interesadas.

Tabla I. Algunas equivalencias propuestas para los cocientes de probabilidad.

\begin{tabular}{ll}
\hline Cociente de probabilidad & Equivalencia verbal propuesta \\
\hline$>10000$ & Muy sólida evidencia para apoyar \\
1000 to 10000 & Evidencia sólida para apoyar \\
100 to 1000 & Evidencia moderadamente sólida para apoyar \\
10 to 100 & Evidencia moderada para apoyar... \\
1 to 10 & Evidencia limitada para apoyar... \\
& \multicolumn{1}{c}{ Hipótesis de la Fiscalía } \\
1 to 0.1 & Evidencia limita contra... \\
0.1 to 0.01 & Evidencia moderada contra... \\
0.01 to 0.001 & Evidencia moderadamente sólida contra \\
0.001 to 0.0001 & Evidencia sólida contra... \\
$<0.0001$ & Evidencia muy sólida contra \\
\hline
\end{tabular}

\section{b) Escala logarítmica del cociente de probabilidad}

Una de las ventajas de esta escala es la comodidad que ofrece para trabajar con números grandes. El problema de esta propuesta podría estar en que algunos logaritmos pueden percibirse menos representativos del grado de solidez de la evidencia 
que comportan. Por ejemplo, el logaritmo 4 puede resultar para un no especialista menos gráfico del concepto de apoyo a la hipótesis de la Fiscalía que un cociente de 10.000 .

Tabla II. Equivalentes logarítmicos de algunos cocientes de probabilidad.

\begin{tabular}{|c|c|c|c|}
\hline Cociente de probabilidad & Equivalencia log & \multicolumn{2}{|c|}{ Equivalencia verbal propuesta } \\
\hline$>10000$ & $>4$ & Muy sólida... & \multirow{5}{*}{$\begin{array}{l}\text { apoyo a la } \\
\text { - hipótesis de } \\
\text { la Fiscalía }\end{array}$} \\
\hline 1000 to 10000 & 3 a 4 & Sólida & \\
\hline 100 to 1000 & 2 a 3 & Moderadamente sólida... & \\
\hline 10 to 100 & 1 a 2 & Moderada... & \\
\hline 1 to 10 & 0 a 1 & Limitada... & \\
\hline 1 to 0.1 & $0 a-1$ & Limitada... & \\
\hline 0.1 to 0.01 & $-1 \mathrm{a}-2$ & Moderada... & apoyo a la \\
\hline 0.01 to 0.001 & $-2 \mathrm{a}-3$ & Moderadamente sólida... & - hipótesis de \\
\hline 0.001 to 0.0001 & $-3 a-4$ & Sólida & \\
\hline$<0.0001$ & $>-4$ & Muy sólida & \\
\hline
\end{tabular}

\subsubsection{Las probabilidades previas}

La propiedad combinatoria, recién comentada, también se puede aplicar a las probabilidades previas. Este concepto se refiere a la cantidad de creencia para la afirmación de la Fiscalía antes de que la evidencia de discurso fuese considerada (previa a la comparación). Para ilustrar el concepto, Rose presenta un ejemplo donde se intercepta una llamada incriminatoria en una casa donde habitaban 5 hombres, incluyendo al sospechoso y que estaban presentes en el momento de la llamada. La probabilidad previa aquí se relaciona con la probabilidad de que el sospechoso hiciera la llamada que es 1/5 ó 0,2 que es el resultado de la aplicación de la fórmula que aparece en la Figura 3. Luego, se le solicita a un perito estimar el LR para la muestra del sospechoso (por ser la única muestra de voz disponible) y éste obtiene un LR global de 20 sobre la base de la evidencia fonética forense. Sólo en base al resultado, esto significa que uno tendría 20 veces más probabilidades de observar el grado de diferencia entre la voz del sospechoso y la voz de la llamada incriminatoria si provinieron del mismo locutor que si fueron emitidas por locutores diferentes. Sin embargo, si se combinan las probabilidades previas con la evidencia de voz $(20 \times 1 / 4=)$ el producto es 5 . 


$$
\mathrm{POA}_{\mathrm{p}}=\frac{p(\mathrm{Ap})}{p(\sim \mathrm{Ap})}
$$

Figura 3. Probabilidades previas para la afirmación de la Fiscalía $A_{p}$ de que dos muestras provienen de la misma voz.

No obstante esta posibilidad y su importancia por la evidente incidencia en el resultado, no ahondaremos más, dado que como reconoce Rose, a pesar de la importancia de las probabilidades previas, al fonetista forense usualmente no se le provee de toda la información necesaria para estimarlas para la evidencia de voz y, por lo tanto, debiera concentrarse sólo en la evaluación del LR para la evidencia de voz. Obviamente, será parte de la responsabilidad del abogado asegurarse de que las probabilidades previas se tomen en cuenta en algún momento en el caso.

Despite the importance of the prior odds, the forensic phonetician is usually not provided with all the necessary information to estimate them for the voice evidence. Some information may be available, for example whether the incriminating telephone calls were made from the accused's house, or their mobile, or whether the accused has been identified by a police officer familiar with his voice. However, as will be explained below, there are also good reasons why the phonetician should insist on not being told such data and should concentrate on assessing the LR for the voice evidence on its own. Obviously, it will be part of the responsibility of legal counsel to ensure that the prior odds are taken into consideration at some point in the case (Rose: 2002: 64).

\subsubsection{La implementación de Morrison (2011), la fórmula del cociente de probabilidad Aitken y Lucy (2004)}

Aitken y Lucy (2004) dedujeron una fórmula del cociente de probabilidad que recoge los conceptos de similitud y tipicidad mencionados. No entraremos en el detalle de la descripción, por ahora sólo nos interesa señalar que Morrison (2007) desarrolló -y aplicó (Morrison, Zhang y Rose, 2011) - un software en Matlab que implementa dicha fórmula y permite aplicarla a los casos forenses.

\section{METODOLOGÍA}

El desarrollo del presente trabajo nos mantuvo ocupados por unos tres meses, si bien nuestras tareas de investigación eran compartidas con otras labores propias del ámbito profesional de cada uno de los autores. Conviene tener presente este 
dato, porque, si bien es cierto algunos procesos pueden variar, normalmente un perito no dispone de mucho tiempo para realizar una comparación científica y la Corte debería ser informada de ese hecho por el bien de la calidad del informe, en caso de ocupar una metodología científica como ésta. A continuación se presenta una síntesis de los pasos que se dieron para la obtención de los resultados del cociente de probabilidad, básicamente: las características esenciales de las muestras en disputa, la elección de la población de referencia y la explicitación de los parámetros acústicos considerados para su análisis.

\subsection{Las muestras comparadas y sus características básicas}

La muestra del sospechoso corresponde a un audio en formato Wav en canal microfónico de 1 minuto y 21 segundos de habla efectiva (es decir, sin silencios), obtenida a partir de una declaración de audiencia del imputado. Por su parte, la muestra incriminatoria corresponde a un audio en formato MP4 de 41 segundos de habla efectiva, extraída del registro de una reunión formal entre dirigentes mapuches y un delegado de la CONADI, cuya duración total es de 58 minutos y 15 segundos. Ambas muestras les fueron llevadas a los primeros dos autores de este trabajo junto con la solicitud de elaborar un informe pericial para determinar si las muestras en disputa procedían o no del mismo locutor, como se señaló al principio.

\subsection{Población de referencia}

La población de referencia debe construirse en base a la voz del sospechoso que sonó similar a la voz incriminatoria para el policía que hizo el reconocimiento (Morrison, Ochoa y Thiruvaran, 2012: 64). Al respecto, conviene tener presente que funcionarios de la Policía de Investigaciones de Chile llevaban unos meses investigando sobre los delitos relacionados con el conflicto mapuche cuando reciben el audio MP4 donde reconocen una de las voces que allí aparecen como una de las que escucharon previamente durante los meses de investigación, que individualiza a Luis Tralcal. En su declaración coinciden en señalar que pudieron reconocer su voz aunque no sabían explicar por qué; simplemente, les resultaba "particular", "como de campo", "cortante". Dado que los funcionarios hicieron la identificación a partir de una grabación microfónica, en un registro campesinomapuche, en estilo de enojo, la población de referencia debería atender a ese perfil, como también responder al perfil sociolingüístico de los policías que efectuaron el reconocimiento.

Combinando lo anterior con los conocimientos de dialectología que nos dicen que los grupos asociados a variables sociales, geográficas y temporales poseen 
rasgos en común frente a otros grupos, se decidió utilizar las muestras de los hablantes del propio audio desde donde se extrae la voz incriminatoria -por ser muestras que proceden de hablantes mapuches y sonar de manera similar a la voz del imputado-; y también se determinó construir una base de datos adicional, con la finalidad de ejemplificar la comparación con una base de datos mayor, para lo cual se estimó que lo más adecuado sería encuestar a hablantes mapuches de la zona para garantizar el parecido con el acusado. Sin embargo, debido a la desconfianza que suscitó entre los lugareños el comentario del propósito científico-forense -que se debía declarar, insoslayablemente, de manera previa a la entrevista ${ }^{5}$ - se tuvo que descartar esta alternativa y en su lugar utilizar muestras extraídas de internet.

Se decidió, entonces, trabajar con dos tipos de muestras diferenciadas, pero complementarias, que servirían a la postre para reforzar cualquier resultado: los hablantes mapuches del audio de la CONADI, ya dado, la más importante; y las muestras extraídas de internet, no disponibles en ese momento, y que tendríamos que buscar, seleccionar y validar perceptivamente por los policías que hicieron el reconocimiento. Ambos tipos de muestras conformarían dos poblaciones de referencia; se trataría de dos sistemas de comparación, independientes que permitirían reforzar el resultado del cociente de probabilidad. A continuación se describen los procedimientos utilizados para la obtención de cada grupo.

\subsubsection{Población de referencia $N^{o} 1$}

La población de referencia No 1 se conformó a partir de las muestras de locutores mapuches presentes en el audio del MP4 que contiene la voz incriminatoria. Para su obtención se aplicó un análisis perceptivo con el objeto de distinguir las voces contenidas en el audio que sonaban distintas entre sí. El audio se segmenta de acuerdo a las intervenciones de los hablantes que se suceden, dando por resultado un total de 219 intervenciones. Para dicho reconocimiento nos hemos basado en aspectos fonéticos y de contenido léxico-semántico. Luego de ello y dejando de lado la voz incriminatoria (51 intervenciones), como la voz del delegado de la CONADI (35 intervenciones) y algunas voces no discriminables (59 intervenciones), se logran distinguir 4 voces diferentes. A continuación se presenta la distribución completa de las voces contenida en el audio reunión:

\footnotetext{
${ }^{5}$ Se siguen los principios éticos que rigen las investigaciones financiadas por el Fondo de Investigación Científica y Tecnológica del Gobierno de Chile (Fondecyt) y que exige el consentimiento informado en la investigación con personas. Ver documentos asociados a su normativa en http:// www.conicyt.cl/fondecyt/2012/10/31/bioetica/.
} 
Tabla III. Voces diferentes presentes en el audio de la reunión de la CONADI.

\begin{tabular}{|c|c|}
\hline Voces de hablantes $(\mathrm{H})$ & No de intervenciones \\
\hline H1 & 6 \\
\hline H2 & 19 \\
\hline Sospechoso. & 51 \\
\hline Delegado de la CONADI & 35 \\
\hline H3 & 39 \\
\hline H4 & 10 \\
\hline No discriminables por ruido, solapamientos y/o escasa duración. & 59 \\
\hline TOTAL & 219 \\
\hline
\end{tabular}

\subsubsection{Población de referencia $N^{\circ} 2$}

Una segunda población estuvo conformada por muestras de locutores mapuches extraídas de internet. Para ello se realizó una búsqueda en radios y TV, dando por resultado 18 direcciones de internet donde aparecían conversaciones entre mapuches, o entre mapuches y no mapuches, a partir de las cuales se seleccionaron 25 locutores, cada uno con una muestra de duración neta de entre aproximadamente 1 y 2 minutos. Para validar el parecido de las muestras de referencia se aplicó un test perceptivo a auditores sin conocimientos lingüísticos, con el fin de emular el perfil de los funcionarios policiales que efectuaron el reconocimiento auditivo que individualizó a Luis Tralcal como sospechoso de los hechos ${ }^{6}$. Sin embargo, la aplicación del test no dio el resultado que se esperaba: una percepción homogénea de semejanza en todos los casos y en el mismo grado que pudiera aproximarse lo más posible a la percepción de los funcionarios policiales que actuaron en el reconocimiento que individualizó a Luis Tralcal; o dicho de otra manera, las muestras de internet no se percibían similares a la voz del sospechoso.

La importancia que reviste la población de referencia nos habría hecho desestimar los datos recogidos, en un caso real, es lo que debiera haber procedido; sin embargo, dado que era una población de prueba, se decidió cambiar el objetivo y mantenerla ${ }^{7}$. Pues bien, la finalidad ya no sería potenciar algún resultado espe-

${ }^{6}$ La razón por la que no se aplicó el test a funcionarios policiales, como una forma de reconstituir de manera ideal la escena auditiva, fue la de que los funcionarios involucrados, en el reconocimiento auditivo que individualizó la voz de la grabación de la CONADI al sospechoso (Tralcal) ya no estaban ubicables en las unidades policiales de origen. Por motivos laborales, los policías van siendo trasladados cada cierto tiempo, lo cual es normal, considerando el tiempo transcurrido (2011-2013).

${ }^{7}$ En esta presentación detallada de las dificultades de los hechos nos inspira el querer mostrar las 
cífico, sino la de observar si el grado de percepción percibida por los auditores, en el caso de que reflejara la realidad de muestras, incidía en los componentes de la relación de probabilidades; puntualmente, ponderando los resultados de "la similitud" entre las muestras comparadas, y afectando con ello de manera errónea los resultados. De modo que la calidad de esta población (percibida de manera no similar a la voz del sospechoso) sería, de manera no programada -al menos inicialmente-, de utilidad para confirmar o no la importancia de reproducir lo más posible el perfil del sospechoso en las muestras de referencia (Cfr. Rose, 2002), hecho que se vio, efectivamente, confirmado, como se muestra más adelante en los resultados.

\subsection{Parámetros acústicos seleccionados para la comparación de las muestras}

Como se ha señalado, la comparación científica bajo el enfoque bayesiano requiere de los datos de la voz incriminatoria, del sospechoso y de una población de referencia, que en su conjunto forman un sistema. Para la obtención de los datos medidos que serían ingresados al sistema de comparación se determinó seleccionar el F2, bajo cuyo término se segmentaron y midieron 50 vocales del fonema /a/ del discurso de cada locutor; en total 1.550 datos, para cuyo análisis se utilizó la herramienta Praat versión 5.3.03, $2011^{8}$.

\subsubsection{Comparación de los datos obtenidos de las muestras en disputa}

En el proceso de determinación del cociente de probabilidad de las muestras del delincuente y del sospechoso se aplicó una doble comparación; la primera se realizó con la población de referencia 1 y la segunda, con la población de referencia 2. Para ello se utilizó el Software de Morrison (2007) que constituye una implementación de la fórmula del cociente de probabilidad de Aitken y Lucy (2004).

\section{RESULTADOS}

Se comparó la muestra del delincuente (extraída de la grabación de una reunión entre dirigentes mapuches y un delegado de la CONADI) y la muestra del sospechoso Luis Tralcal (obtenida de una grabación de una declaración de Audiencia).

verdaderas dificultades a las que se enfrenta un analista científico y las consecuencias de su accionar en la realidad; como asimismo, la actitud que también debe adoptar el científico en su Informe de manera que el juez pueda, entre dos o más informes de análisis del mismo tipo, valorar la calidad y decidir en consecuencia.

${ }^{8}$ También se midió el F1 del fonema /a/ y un análisis idéntico se aplicó al fonema /e/, con el fin de servir para trabajos futuros. 
Primero, se utiliza como población de referencia 4 voces que aparecen en el mismo audio de la reunión de los dirigentes de la CONADI. Y, posteriormente, se utiliza como población de referencia un conjunto de muestras de 25 hablantes mapuches, obtenido desde internet.

Previo al detalle de los resultados, conviene recordar que un cociente menor a la unidad se traduce en locutores diferentes.

\subsection{Resultados para la población de referencia 1 (audio CONADI)}

Se aplican dos ejercicios de comparación: uno donde la muestra del sospechoso (Tralcal) se compara con la muestra del delincuente (NN); y otro, donde se compara la muestra del sospechoso (Tralcal) con cada una de las muestras del resto de los hablantes del audio de la CONADI (H1, H2, H3 y H4). En cada caso, la población de referencia son todos los hablantes del audio, excepto el considerado "delincuente" que está siendo comparado con el sospechoso. El objetivo, en este caso, es descartar o afirmar que alguno de los otros hablantes del audio de la grabación de la CONADI fuese Tralcal. Ver resumen gráfico en las Tablas IV y V que se presentan a continuación.

Tabla IV. Esquema del primer ejercicio de comparación de la muestra del sospechoso (Tralcal) con la muestra del delincuente (NN).

\begin{tabular}{|c|c|c|}
\hline Muestra del sospechoso & Muestra del delincuente & Población de referencia \\
\hline & & $\mathrm{H} 1$ \\
\hline Tralcal & $\mathrm{NN}$ & $\mathrm{H} 2$ \\
\hline & & $\mathrm{H} 3$ \\
\hline & & $\mathrm{H} 4$ \\
\hline
\end{tabular}

Tabla V. Esquema del segundo ejercicio de comparación de la muestra del sospechoso (Tralcal) con cada una de las muestras del audio de la CONADI ( $\mathrm{H} 1, \mathrm{H} 2, \mathrm{H} 3$ y $\mathrm{H} 4)$.

\begin{tabular}{|c|c|c|}
\hline Muestra del sospechoso & Muestra del delincuente & Población de referencia \\
\hline Tralcal & $\begin{array}{l}\mathrm{H} 1 \text {, luego } \mathrm{H} 2, \mathrm{H} 3 \\
\text { y finalmente } \mathrm{H} 4 .\end{array}$ & $\begin{array}{c}\text { Todas las muestras del audio, } \\
\text { menos la considerada como } \\
\text { muestra del delincuente: } \\
\text { H2, H3, H4, NN; luego H3, } \\
\text { H4, NN, H1 y así sucesivamente. }\end{array}$ \\
\hline
\end{tabular}




\subsubsection{Resultado primer ejercicio de comparación de la muestra del sospechoso (Tralcal) con la muestra del delincuente (NN).}

a) Muestra sospechoso vs muestra del delincuente: Cociente de probabilidad= 0.2653 . El resultado específico obtenido significa que las diferencias observadas tendrían $(1 / 0,2653=)$ 3,76 veces más probabilidades de ser observadas, asumiendo locutores diferentes. Si se asume alguna escala verbal o logarítmica, el resultado numérico se podría traducir como un moderado apoyo a la hipótesis de la Defensa o evidencia moderada contra la hipótesis de la Fiscalía. En todo caso, lo que hay que subrayar es que el resultado del cociente nos dice que el sospechoso no es el delincuente.

\subsubsection{Resultados del segundo ejercicio para la comparación de la muestra del sospechoso (Tralcal) con cada una de las muestras del audio de la CONADI (H1, H2, H3 y H4).}

a) Muestra sospechoso vs muestra de otro hablante del audio (H1) como muestra del delincuente: Cociente de probabilidad $=0,0307$. El resultado obtenido significa que las diferencias observadas tendrían $(1 / 0,0307=)$ 32,57 veces más probabilidades de ser observadas, asumiendo locutores diferentes: moderado apoyo a la hipótesis de la Defensa o evidencia moderada contra la hipótesis de la Fiscalía.

b) Muestra sospechoso vs muestra de otro hablante del audio (H2) como muestra del delincuente: Cociente de probabilidad $=0,0402$. El resultado obtenido significa que las diferencias observadas tendrían $(1 / 0,0402=)$ 24,87 veces más probabilidades de ser observadas, asumiendo locutores diferentes: moderado apoyo a la hipótesis de la Defensa/evidencia moderada contra la hipótesis de la Fiscalía.

c) Muestra sospechoso vs muestra de otro hablante del audio (H3) como muestra del delincuente: Cociente de probabilidad $=0,0330$. El resultado específico obtenido significa que las diferencias observadas tendrían $(1 / 0,0330=) 30,30$ veces más probabilidades de ser observadas, asumiendo locutores diferentes: moderado apoyo a la hipótesis de la Defensa/evidencia moderada contra la hipótesis de la Fiscalía.

d) Muestra sospechoso vs muestra de otro hablante del audio (H4) como muestra del delincuente: Cociente de probabilidad $=2,5370 \mathrm{e}-006\left(2,5370 \times 10^{-6}\right)$ 0,000002537 . El resultado obtenido significa que las diferencias observadas tendrían $(1 / 0,000002537=)$ 394166,33 veces más probabilidades de ser observadas, asumiendo locutores diferentes: muy sólido apoyo a la hipótesis de la Defensa/evidencia muy sólida contra la hipótesis de la Fiscalía. 
En síntesis: el sospechoso (Tralcal) no estaba o no habló en el audio de la reunión.

\subsection{Resultados para la población de referencia No 2 (audios de Internet)}

Se compara, nuevamente, la muestra del sospechoso (Tralcal) con la muestra del delincuente $(\mathrm{NN})$, pero esta vez teniendo como población de referencia las muestras de 25 hablantes mapuches extraídas de internet. El propósito es observar si un perfil deliberadamente no ajustado a las características del sospechoso incide en los componentes de la relación de probabilidades (Cfr. Rose, 2002); puntualmente, ponderando los resultados de "la similitud" entre las muestras comparadas, y afectando con ello de manera errónea los resultados.

a) Muestra sospechoso vs muestra del delincuente: Cociente de probabilidad =0,5101. El resultado específico obtenido significa que las diferencias observadas tendrían $(1 / 0,5101=)$ 1,960 veces más probabilidades asumiendo locutores diferentes. En este caso, la probabilidad de apoyo a la Defensa se reduce, respecto de la comparación anterior que tenía en cuenta la población de los 4 hablantes del audio de la CONADI, pero igualmente nos dice que se trata de locutores diferentes.

Se trata, hasta cierto punto de un resultado esperable, dadas las características de las muestras con que se construyó la población de referencia $2^{9}$. Al respecto, conviene recordar, por una parte, que las muestras de internet no fueron percibidas de manera homogénea tras la aplicación del test, no obstante se decidió utilizarlas sin cambiar nada con el propósito de observar la incidencia de su calidad en los resultados; y, por otra parte, que una diferencia entre la población de referencia y del sospechoso puede afectar artificialmente "la tipicidad" y ponderar, erróneamente, por consiguiente, "la similitud" de las muestras comparadas (Cfr. Rose, 2002). Es un hecho que habrá que tener en cuenta; la población de referencia debe ser lo más parecida posible a la muestra del sospechoso y esto obliga a trabajar con poblaciones ad hoc.

\section{CONCLUSIONES}

Con el objeto de ilustrar mejor nuestros resultados y sopesar sus implicancias teóricas y prácticas, supongamos que en el caso real del 2011 el científico forense

\footnotetext{
${ }^{9}$ Ver detalles en apartado Metodología de este mismo trabajo.
} 
hubiese ocupado el mismo enfoque y procedimientos, obteniendo en consecuencia los mismos resultados reseñados, ¿cuál debiera haber sido su declaración? Una forma posible podría haber sido ésta:

"En relación a la comparación solicitada por la Defensa sobre si las muestras de la voz del sospechoso (Tralcal) y del delincuente (cuya identidad se desconoce) proceden o no de la misma persona y basándome en un enfoque bayesiano, conocido como cociente de probabilidad, mi evaluación de las diferencias observadas del F2 del fonema /a/ entre ambas muestras da como resultado un cociente de probabilidad igual a 0.2653 , donde se establece claramente que un cociente menor a la unidad se traduce en locutores diferentes o que la probabilidad para la hipótesis de la Defensa es mayor".

La importancia de un análisis y expresión de resultados como éste salta a la vista por la objetividad e inteligibilidad que ofrece a la Corte, permitiéndole al científico efectivamente colaborar en la decisión -que no pronunciarse sobre inocencia y menos culpabilidad del imputado-que le corresponde al tribunal, el cual maneja otros datos y en virtud de lo cual un informe como éste orienta su acción.

No obstante lo reseñado, las ventajas de una metodología como la descrita y los resultados obtenidos para este caso en particular, deseamos dejar en claro que la aplicación de una metodología como ésta exige todavía un tiempo razonable de ejercitación y de su correspondiente difusión dentro de la comunidad científica antes de ser utilizada de manera corriente en la Corte. Esto debe derivar, al menos, en dos acciones básicas: a) seleccionar casos reales de referencia de investigación, porque son éstos los que dejan ver las verdaderas dificultades teóricas y prácticas que debe sortear el analista en terreno; y b) trabajar con grandes y diversas cantidades de datos de laboratorio, probando el rendimiento de parámetros acústicos, aislados y/o combinados.

\section{REFERENCIAS}

Aitken, C.G.G. y Lucy, D. (2004). Evaluation of trace in the form of multivariate data. Appplied Statistics, 53, 4, 109-122.

Cerda San Martín, Rodrigo. (2008). Valoración de la Prueba. Sana Crítica. Santiago: Librotecnia.

Champod, C. and Evett, I. (2000). Commentary on Broeders (1999), FL $7 / 2,238-43$.

Evett, I. W. (1991). Interpretation: A personal odyssey. En C.G.G Aitken y D.A. Stoney (eds.). The use of statistics in forensic science (pp. 9-22). 
Chichester: Ellis Horwood.

Informe Pericial de Temuco. (2011). Causa ROL No 158-2011 del Tribunal Oral en lo penal de Temuco por los delitos de AMENAZA TERRORISTA Y OTROS. Elaboración y exposición en juicio oral del Informe Pericial de Fonética Forense por Claudia Rosas y Jorge Sommerhoff, ofrecida y presentada por la Defensa con fecha 28 de septiembre de 2011.

Morrison, G. S. (2007). Matlab implementation of Aitken \& Lucy's (2004) forensic likelihood-ratio software using multivariate-kernel-density estimation. [Software]. Disponible en http://geoff-morrison.net. Consulta: 10.03.2014.

Morrison, G.S., Zhang, C. y Rose, P. (2011). An empirical estimate of the precision of likelihood ratios from a forensic-voice-comparison system. Forensic Science International, 208, 59-65.

Morrison, G. S., Ochoa, F. y Thiruvaran, T. (2012). Database selection for forensic voice comparison. En International Speech Communication Association (pp. 62-77). Presentado en Proceedings of Odyssey 2012: The Language and Speaker Recognition Workshop, Singapore: 15-28 de junio.

Robertson, B. y G.A. Vignaux. (1995). Interpreting evidence. UK: Wiley, Chichester.

Rose, P. (2002). Forensic speaker identification. London: Taylor and Francis. 